\title{
RESOLUTIONS
}

\author{
Resolution No. 1
}

\section{THE SYMPOSIUM}

notes and welcomes the following recommendation of the ICG and urges the participants and their organizations to actively cooperate:

"the Geodynamic Commission notes that recent evidence regarding the structure and evolution of satellite and planetary interiors and of their motions in space is being provided by use of radio and optical methods, as well as by space technology. Theories about the evolution of the Earth should therefore be tested by these data and full interchange of information and discussion between scientists working in these fields is essential".

\section{Resolution No. 2}

THE SYMPOSIUM

considering the results achieved by means of classical astronomical observations in the determination of polar motion and variations in the rotational velocity of the Earth and the necessity of extending the data in a homogeneous system into the future; the higher precision which may be achieved by new techniques such as laser measurements of distances to the Moon or artificial satellites, Doppler observations, VLBI, etc.

recommends:

(1) That the classical instruments (astrolabes, photo-electric transits, PZT's, VZT's, etc.) should continued to be used (or their use should be resumed) to the maximum of their capabilities at highest standards of maintenance, in conjunction with the necessary observational programmes and instrumental improvements in order to continue acquisition of homogenous data.

(2) That the new techniques be further developed and, as soon as satisfactory precision is in sight, coordinated long term programmes using these new techniques be planned;

that the data processing procedures be fully described and made available to the scientific community;

that the results obtained by these new techniques be made available in a homogeneous system, implying also that whenever the data processing procedures are changed previously taken observations be reduced.

(3) That the results obtained by classical and new techniques be compared over a sufficiently long period to permit investigations on secular and long periodic phenomena; 
that selected new stations employing the new techniques should be located at astronomical and/or geophysical observatories, or be equipped with appropriate geophysical instruments.

\section{Resolution No. 3}

THE SYMPOSIUM

considering the level of accuracy already achieved by laser ranging to artificial satellites and the significance of this technique to astronomy and the Earth sciences,

recommends that in order to take full advantage of this technique and to assure accurate data acquisition over long periods of time, satellites equipped with laser corner reflectors, to be launched in the future for astronomical and geophysical purposes, should be isotropic and have high altitudes and high mass to area ratios to reduce perturbations; should be put into high inclination orbits to insure world wide tracking coverage; and should have a shape and corner reflector arrangement such that its center of mass can be accurately indentified.

\section{Resolution No. 4}

THE SYMPOSIUM

considering the importance of the elastic properties of the Earth and noting the very high quality local parameters obtainable by means of modern instruments,

recommends that appropriate institutions should establish Earth tidal stations, especially in the southern hemisphere, utilizing the most recent measurement systems, including gravimeters, horizontal pendulums, extensometers and lasers, and that these stations be located over as wide an area as possible.

\section{Resolution No. 5}

THE SYMPOSIUM

considering that a PZT is already in operation at Mizusawa, and that, in accordance with the resolutions adopted by the IAU in Prague and by the IUGG in Lucerne, the installation of a PZT at Kitab has been decided, and that similar installations are under active consideration at Ukiah and Gaithersburg,

recommends that in order to complete the five ILS stations with PZT's, the new Cagliari observatory should also be provided with a PZT. The cooperation of the appropriate Italian authorities in this matter is hoped for.

\section{Resolution No. 6}

The participants in Symposium No. 48 of the International Astronomical Union, Morioka, May 1971, wish to express their gratitude and appreciation to 
the Ministry of Education, the Science Council of Japan, the Astronomical Society of Japan, the Geodetic Society of Japan

for their invitation to hold the meetings in Morioka, and for their provision of the necessary facilities;

also to the Local Organizing Committee; the Chairman, Dr. Okuda, Director of the Observatory of Mizusawa; the Secretary, Dr. Yumi, Director of the International Polar Motion Service; and to all the members and helpers for their excellent arrangements and their willing assistance;

also to the Governor of Iwate Province, and the Mayors of Morioka and Mizusawa City, for their warm welcome and generous hospitality. 